\title{
Alteraciones dermatológicas en pacientes con anemias carenciales
}

\author{
Oscar Ruiz 1,2, Luz Bardales 2, David Díaz 2, Carlos Galarza 1,3, Carlos Delgado 1,2, \\ Oscar Castillo ${ }^{2}$, Manuela Marangoni ${ }^{4}$, Carlos Montenegro ${ }^{1}$
}

Resumen

Palabras clave

\begin{abstract}
Objetivos: Determinar la frecuencia de las alteraciones dermatológicas relacionadas a las anemias carenciales, así como el tipo y grado de severidad de la anemia. Materiales y Métodos: Se estudió 100 pacientes mayores de 18 años con anemia carencial atendidos en el Hospital Dos de Mayo, entre enero y noviembre de 2004. A todos se les realizó hemograma, constantes corpusculares y determinación sérica de hierro, ferritina, ácido fólico y vitamina B12. Resultados: En orden de frecuencia, el tipo de anemia fue ferropénica 45\%, carencial mixta (ferropénica y megaloblástica)35\% y megaloblástica 20\%. Las manifestaciones dermatológicas más frecuentes fueron palidez $100 \%$, glositis atrófica $81 \%$, queilitis angular 60\%, coiloniquia 33\%, escleras azuladas $30 \%$, fragilidad capilar 29\%, fragiliadad ungueal 29\%. El grado de anemia más frecuente fue el severo, $75 \%$. Conclusiones: El $80 \%$ de pacientes presentó más de cuatro alteraciones dermatológicas: palidez, glositis atrófica, queilitis angular, coiloniquia y escleras azuladas. La anemia carencial más frecuente fue la ferropénica, con $45 \%$.
\end{abstract}

Anemias nutricionales; anemia ferropriva; piel; transtornos nutricionales.

\begin{abstract}
Dermatologic alterations in nutritional anemias Abstract

Objectives: To determine the frequency of anemia-related dermatological abnormalities and severity. Materials and Methods: A sample of 100 subjects older than 18 years with a diagnosis of nutritional anemia who were admitted at 2 de Mayo Hospital between January and November, 2004 was studied. In each subject we measured total CBC and serum iron, ferritin, folate, and cobalamin levels. Results: The type of anemia was iron deficiencyin 45\%, mixed anemia(iron deficiency and megaloblastic anemia) in 35\%, and megaloblastic anemia in $20 \%$. Dermatological abnormalities were pallor (100\%), atrophic glossitis (81\%), angular cheilitis (60\%), koilonychia (33\%), tinyblue sclera (30\%), thinning hair (29\%), thinning nails (29\%); $75 \%$ of the cases were determined as having severe anemia. Conclusions: The nutritional anemia most frequently found was iron deficiency (45\%). About $80 \%$ of subjects showed more than four dematological abnormalities: pallor, atrophic glossitis, angular cheilitis, koilonychia and tiny-blue sclera.
\end{abstract}

1 Instituto de Investigaciones Clínicas. Facultad de Medicina, UNMSM. Lima, Perú.

Servicio de Hematología, Hospital Nacional Dos de Mayo. Lima, Perú.

3 Servicio de Dermatología, Hospital Nacional Dos de Mayo. Lima, Perú.

4 Departamento de Enfermería, Hospital Nacional Dos de Mayo. Lima, Perú.
Keywords: Nutritional anemias; anemia, iron-deficiency; skin; nutrition disorders.

\section{INTRODUCCIÓN}

Las anemias carenciales se deben a la falta de oligoelementos, como hierro y/o vitaminas, como el ácido fólico y la vitamina B12 $\left({ }^{1,2}\right)$. Estos elementos involucrados en la patogenia son indispensables para la formación de tejidos con alto recambio celular, como es el caso de la piel, las mucosas y las faneras $\left({ }^{1,3,4}\right)$.

La piel, mucosas y faneras son los componentes que más impactan cuando se realiza un examen clínico a una persona con el diagnóstico de anemia carencial. Se sabe que en la anemia ferropénica se pueden presentar queilitis angular, escleras azuladas, fragilidad capilar, coiloniquia, glositis atrófica, etc $\left({ }^{1,4,5}\right)$. Así mismo, en la anemia megaloblástica es frecuente el hallazgo de glositis atrófica, ictericia y sequedad de la piel $\left({ }^{1,2,6}\right)$. 
Las anemias carenciales son más prevalentes en los países en vías de desarrollo, como es el caso del Perú, pues guardan estrecha relación con la capacidad económica de la población y su acceso a los servicios básicos de salud $\left({ }^{1,2}\right)$. Los niveles de pobreza en el Perú afectan a $52 \%$ de su población; por lo tanto, los peruanos son vulnerables a la presentación de anemias carenciales y las alteraciones dermatológicas debidas a este tipo de anemias.

El objetivo de este trabajo es describir las alteraciones dermatológicas más frecuentes en los cuadros de anemia carencial.

\section{MATERIALES Y MÉTODOS}

Se realizó un estudio observacionaltransversal.

Se procedió a la determinación de hemograma, constantes corpusculares, hierro sérico, ácido fólico, vitamina B12 y ferritina sérica en 100 pacientes, atendidos en el Hospital Nacional Dos de Mayo, entre enero y noviembre de 2004. Se incluyó pacientes mayores de 18 años con palidez de piel y mucosas debida a anemia. Se excluyó a pacientes que usaban de suplemento de hierro, vitamina B12 y ácido fólico, tres meses antes del estudio.

Se consideró valores normales los siguientes:

- Hemoglobina:

Varones: 13 a $17 \mathrm{mg} / \mathrm{dL}$

Mujeres: 12 a $16 \mathrm{mg} / \mathrm{dL}$

- Ferritina sérica

Varones y mujeres: 13 a $400 \mathrm{mg} / \mathrm{dL}$

- Vitamina B12

Varones y mujeres: 240-900 pg/mL

- Ácido fólico:

Varones y mujeres: 4,9 a 19,9 $\mathrm{ng} / \mathrm{mL}\left({ }^{7}\right)$

- Grado de anemia (ambos sexos)

(hemoglobina en $\mathrm{g} / \mathrm{dL}$ )

$\begin{array}{ll}\text { Leve } & 10 \text { a } 11,9 \text { (mujer) } \\ & 10 \text { a } 12,9 \text { (varón) } \\ \text { Moderada } & 7 \text { a } 9,9 \\ \text { Severa } & <7 \quad\left({ }^{2}\right)\end{array}$

\section{RESULTADOS}

De la población estudiada (100 personas), 60 fueron mujeres y 40 varones; la edad promedio fue 37 años, con un rango de 18 a 70 años. Los resultados finales según tipo de anemia fueron (Figura 1): ferropénica $(45 \%)$, carencial mixta $(35 \%)$, megaloblástica $(20 \%)$. Dentro de las anemias carenciales mixtas, el componente megaloblástico se debió a la disminución del folato $(25 \%)$ y a la carencia de vitamina B12 $(10 \%)$. En relación a las anemias megaloblásticas, la carencia de B12 se presentó en $15 \%$ y la disminución del folato en $5 \%$.

El grado de anemia fue (Figura 2) severo $(75 \%)$, moderado $(20 \%)$ y leve $(5 \%)$.

Las alteraciones dermatológicas en orden de frecuencia fueron (Figura 3): palidez $100 \%$, glositis atrófica $(81 \%)$ (Figura 4), queilitis angular (60\%) (Figura 5), coiloniquia (33\%) (Figura 6), escleras azuladas $(30 \%)$, fragilidad capilar y fragilidad ungueal (cada una con 29\%) y, finalmente, ictericia, con $23 \%$ del total. El

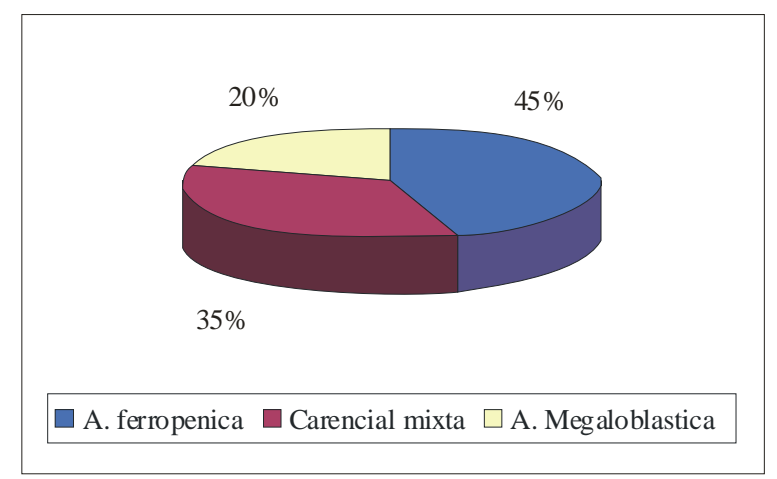

Figura 1. Tipos de anemia. 


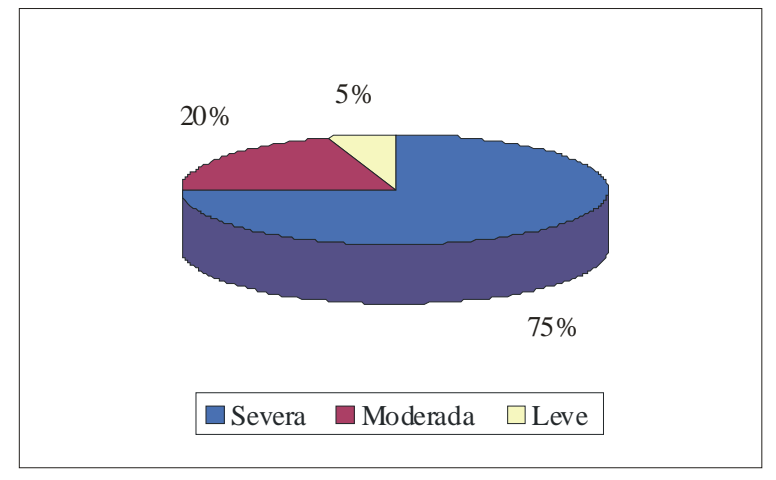

Figura 2. Grado de anemia.

$80 \%$ de los pacientes presentó más de tres manifestaciones dermatológicas del síndrome anémico.

\section{DISCUSIÓN}

Los actuales niveles de pobreza en el país $(52 \%)$ son el terreno ideal para la presentación de anemias carenciales, pues además del poco acceso de la población a los alimentos necesarios para tener un estado nutricional adecuado, se agrega la falta de servicios básicos de salud $\left({ }^{1,2}\right)$.

La piel y las mucosas se constituyen en la parte del organismo que puede expresar

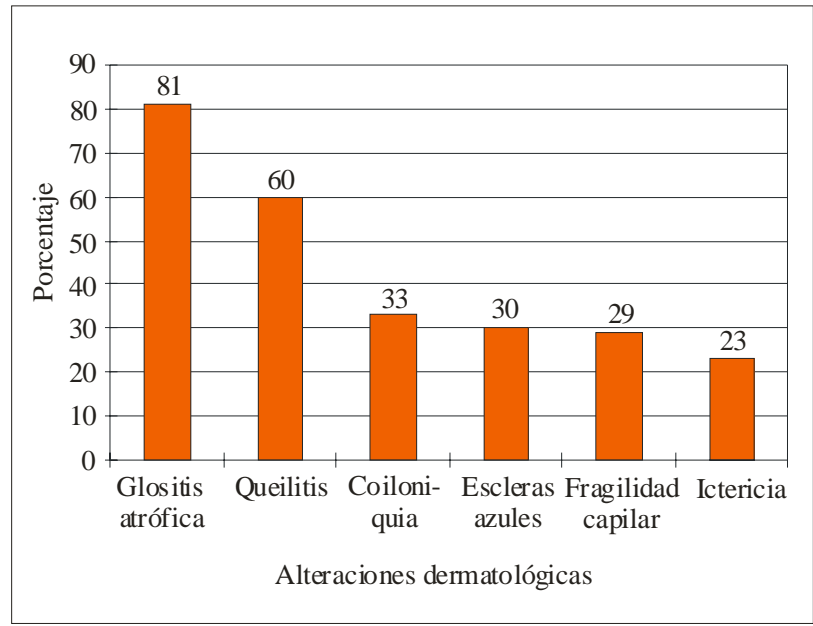

Figura 3. Alteraciones dermatológicas según frecuencia. las consecuencias de las anemias carenciales $\left({ }^{1,2,3,6,8}\right)$. Así, por ejemplo, en la falta de hierro es conocida la presentación de coiloniquia y escleras azules $\left({ }^{1,2,4,5,7}\right)$ y en anemia megaloblástica es frecuente la aparición de glositis atrófica e ictericia $\left({ }^{1,8,9,10}\right)$. Estas manifestaciones dermatológicas se deben a que el hierro, el folato y la vitamina B12 son necesarios para la formación de tejidos de recambio celular elevado $\left({ }^{1,8,9,10,11}\right)$.

Al observar la incidencia de anemia carencial por sexo, se evidencia que ésta es más frecuente en mujeres. Este hecho confirma lo descrito en la literatura, pues tanto los niños y las mujeres en edad fértil son considerados como grupos de alto riesgo. En el caso de las mujeres, la fisiopatología de la anemia involucra tanto la falta de aporte (gestación y nivel socioeconómico deprimido) como las pérdidas debidas a los ciclos menstruales $(1,3,4,7)$.

El hallazgo de más de cuatro alteraciones dermatológicas en $80 \%$ de pacientes se puede deber a que $75 \%$ de estos presentó anemia severa (hemoglobina $<7 \mathrm{~g} / \mathrm{dL}$ ). Este hecho podría ser un índice del poco aporte, tanto de hierro como de ácido fólico y vitamina $\mathrm{B} 12$, que recibe la población en su dieta habitual. Además, se debe tener en cuenta que las personas, sobre todo del

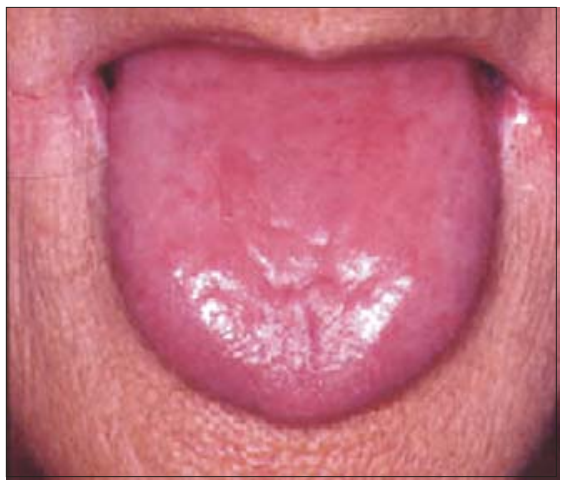

Figura 4. Nótese la evidente glositis atrófica. 


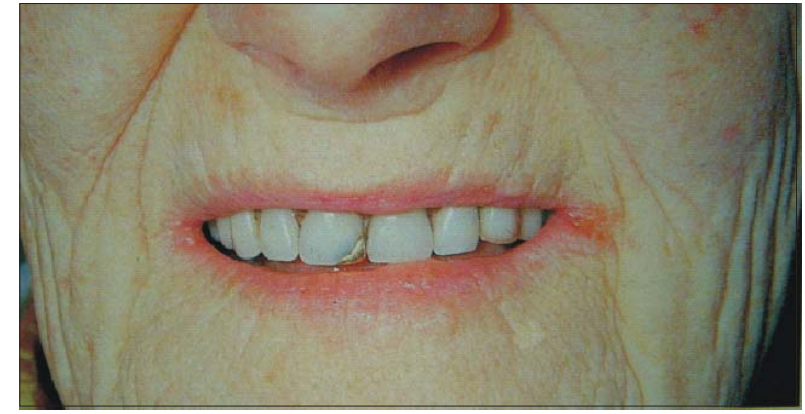

Figura 5. La foto muestra queilitis angular bilateral.

estrato socioeconómico $\mathrm{E}$, tienen poco acceso a los servicios básicos de salud, como son el agua potable y desagüe, por lo que la incidencia de parasitosis intestinal puede ser alta en este grupo.

Las manifestaciones más frecuentes como glositis atrófica, queilitis comisural y coiloniquia- se deben, además de la severidad de la anemia, a la cronicidad de la misma $\left({ }^{9}\right)$.

Al analizar el componente megaloblástico presente en las anemias carenciales mixtas, se observa que es más frecuente la carencia de ácido fólico; esto se puede deber a que los requerimientos diarios son mayores que las de vitamina B12. El papel del ácido fólico es muy importante en la formación de nuevas células y sobre todo en los tejidos que tienen un alto recambio celular, como son la piel y las mucosas.

Los hallazgos del presente estudio pretenden alertar a los médicos generales, dermatólogos y ginecólogos, pues muchas veces son ellos quienes en primera instancia atienden a los pacientes con anemias carenciales. Asimismo, el Ministerio de Salud debe tomar las medidas correctivas necesarias para disminuir la prevalencia de este tipo de anemias, sobretodo en las poblaciones más susceptibles, como son los niños y las mujeres en edad fértil.

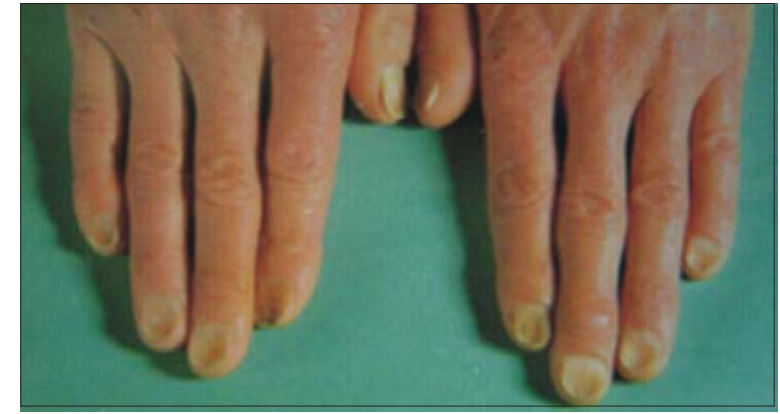

Figura 6. Obsérvese coiloniquia en ambas manos.

\section{REFERENCIAS BIBLIOGRÁFICAS}

1. Lichtman MA, Beutler E, Kipps TJ, Williams WJ. Williams Manual of Hematology. 6th ed. New York, NY: McGrawHill; 2003.

2. Hernández-Nieto L. Protocolos. Anemia. Madrid: Idepsa; 1990.

3. Hernández-Nieto L, Hernández-García MT. Enfermedades del sistema eritrocitario. Anemia posthemorrágica. Anemias por déficit y/o trastorno metabólico de hierro. En: Farreras P, Rozman C (eds.). Medicina Interna Vol II. Barcelona: Doyma; 1992. p. 1617-22.

4. Parsons S, Fleming MD, Nathan DG, Andrewas NC. Iron deficiency anemia associated with an error of iron metabolism in two siblings: a thirty year follow up. Hematology. 1996;1:65-73.

5. Cook JD. Clinical evaluation of iron deficiency. Semin Hematol. 1982;1:6-21.

6. Chanarin I. The megaloblastic anemias. 3th ed. Oxford: Blackwell Scientific; 1990.

7. Crosby WH. The rationale for treating iron deficiency anemia. Arch Int Med. 1984;144:471-3.

8. Herbert V. Vitamin B12. En: Ziegler EE, Filer Lj, eds. Present knowledge in nutrition. 7th ed. Washington, DC: International Life Sciences Institute (ILSI) Press.; 1996. p. 191-205.

9. Castle WB. The Conquest of pernicious anemia. En: Wintrobe MM (ed.). Blood, pure and eloquent. New York: McGraw Hill; 1980.

10. Crowe SF, Ross CK. Effect of folate deficiency and folate and B12 excess on memory functioning in young chicks. Pharmacol Bichem Behav. 1997;56:189-97.

11. Herbert V, Das K. Folic acid and vitamin B12. En: Shils ME, Olson JA, Shike M (eds). Modern nutrition in health and disease ( ${ }^{\circ}$ ed.). Washington: Lea \& Febiger; 1994. p. 402-25.

Manuscrito recibido el 15 de febrero de 2006 y aceptado para publicación el 10 marzo de 2006.

Correspondencia: Dr. Oscar Ruiz, Franco

Instituto de Investigaciones Clínicas

Hospital Nacional Dos de Mayo. Av. Grau cuadra 13.

Parque Historia de la Medicina. Lima 1, Perú.

Correo-e: osma@ec-red.com 